\title{
Vestibular Control of Intermediate- and Long-Term Cardiovascular Responses to Experimental Orthostasis
}

\author{
G. RAFFAI ${ }^{1}$, C. CSEKÖ ${ }^{2}$, G. NÁDASY ${ }^{1}$, E. MONOS $^{1}$ \\ ${ }^{1}$ Institute of Human Physiology and Clinical Experimental Research, Semmelweis University, \\ Faculty of Medicine, Budapest, Hungary, ${ }^{2}$ Department of General Pharmacology Gedeon Richter \\ Ltd., Budapest, Hungary
}

Received September 27, 2008

Accepted January 28, 2009

On-line February 27, 2009

\section{Summary}

Sustained orthostasis elicits the elevation of arterial blood pressure (BP) via sympathetic activation in conscious Wistar rats for at least 2 hours. We tested the hypothesis whether vestibular apparatus plays a role in BP and heart rate (HR) control in response to prolonged gravitational stress. $\mathrm{BP}$ and $\mathrm{HR}$ responses to $45^{\circ}$ head-up for either 2 or 24 hours were monitored by telemetry. Vestibular lesions (VL) were performed by a modified microsurgical-chemical technique. Horizontal BP and HR were not influenced by VL preceding 2-hour tilt. VL abolished the sustained 2-hour BP response to head-up tilt $(8.3 \pm 0.9 \mathrm{~mm} \mathrm{Hg}$ relative to horizontal values) while suppressed HR transiently only. VL eliminated diurnal BP fluctuations and decreased HR in horizontal position for 24 hours. Head-up tilt for 24 hours increased BP and $\mathrm{HR}$ progressively in intact animals, raising their daily average value by $5.6 \pm 0.7 \mathrm{~mm} \mathrm{Hg}$ and $22.2 \pm 6 \mathrm{BPM}$, respectively. VL resulted in an initial $\mathrm{BP}$ rise followed by progressive $\mathrm{BP}$ reduction in response to long-term head-up tilt $(4 \pm 2.2 \mathrm{~mm} \mathrm{Hg})$ without eliminating the tachycardia (34.4 \pm 5.4 BPM). Thus, blockade of labyrinthine inputs attenuates the BP responses elicited by both intermediate and long-term gravitational stress of orthostatic type. However, other sensory inputs derived from non-vestibular cues (e.g. proprioceptive, visual, visceral, cutaneous etc.) seem to be effective enough to maintain BP normal.
\end{abstract}

\section{Key words}

Arterial blood pressure - Cardiovascular control - Heart rate • Orthostasis • Vestibular lesion

\section{Corresponding author}

Gábor Raffai, Institute of Human Physiology and Clinical Experimental Research, Semmelweis University, Tüzoltó utca 3747, H-1094, Budapest, Hungary. Fax.: (36-1) 334-3162. E-mail: raffai@elet2.sote.hu

\section{Introduction}

Activation of gravity-related reflexes linked to the changes in body position and/or other gravitational stress can be induced by several receptor mechanisms in addition to the well-known baroreceptor system. Labyrinthine, proprioceptive, visual, visceral and cutaneous inputs, which converge on the neurons of the central neural vestibular system have been implicated in cardiovascular adaptation (Balaban and Beryozkin 1994, Jian et al. 1999, Jian et al. 2002, Kerman et al. 2000, Mori et al. 2005, Ray and Carter 2003, Voustianiouk et al. 2006, Yates et al. 2000, Yates and Bronstein 2005). These inputs, when transmitted to the circulatory control area of the brainstem, may influence sympathetic output, contributing to the complex cardiovascular control that compensates for imbalances caused by body fluid shift and blood redistribution during postural changes (Balaban and Beryozkin 1994, Doba and Reis 1974, Jian et al. 1999, Kerman et al. 2000, Ray and Bronstein 2003, Yates et al. 2000, Yates and Bronstein 2005). Among these mechanisms, the operation of the vestibulosympathetic reflex seems to be the most significant in gravitational adaptation of both humans (Hume and Ray 1999, Monos and Lóránt 1998, Ray et al. 1998a,b, Ray 2000, Ray 2001, Shortt and Ray 1997, Voustianiouk et al. 2006, Yates et al. 2000, Yates and Bronstein 2005) and animals (Doba and Reis 1974, Gotoh et al. 2004, Jian et al. 1999, Kerman et al. 2000, Monos and Lóránt 1998, Mori et al. 2005, Tanaka et al. 2006, Wilson et al. 2006, Woodring et al. 1997, Yates et al. 2000, Yates and Bronstein 2005). 
The vestibulosympathetic reflex is supposed to help to maintain orthostatic tolerance in humans via activation of sympathetic efferents mediating short-term changes in blood pressure during rapid changes of body and/or head position (Ray 2000, Ray and Carter 2003, Shortt and Ray 1997, Voustianiouk et al. 2006, Yates et al. 2000, Yates and Bronstein 2005). Vestibulosympathetic reflex also seems to be working independently from the baroreflex when adjusting the cardiovascular system to postural changes and their effect is additive with regard to muscle sympathetic nerve activity (Ray 2000, Shortt and Ray 1997). Moreover, there are data providing evidence that otolith organs (Ray 2000, Shortt and Ray 1997), but not the semicircular canals (Ray et al. 1998b), neck afferents (Ray et al. 1998a), non-specific pressure receptors in the head (Hume and Ray 1999), or skeletal muscle receptors (Ray 2001) are the major sources of sympathetic nerve activation in humans.

Several experimental findings have proven the significance of vestibular control of arterial blood pressure in different animal models during postural or gravitational changes. For example, in the head-up tilted position, arterial blood pressure (Doba and Reis 1974, Woodring et al. 1997) and vascular resistance (Doba and Reis 1974) cannot be maintained in either anesthetized or paralyzed cats (Doba and Reis 1974), or in cats subjected to extensive denervations (Woodring et al. 1997) following transsection of the vestibular nerves. Similarly, bilateral lesions of vestibular receptors (Jian et al. 1999) or the vestibular nucleus (Mori et al. 2005) also compromise orthostatic tolerance in awake cats during $60^{\circ}$ nose-up tilt. During microgravity and hypergravity exposures, both the acute pressor and the renal sympathetic nerve activity responses were attenuated by vestibular lesion (Gotoh et al. 2004, Tanaka et al. 2006).

Most of the studies above investigated the role of the vestibular system in immediate and short-term ( $\leq 5 \mathrm{~min}$ ) dynamic responses. However, the role of the vestibular system in prolonged, intermediate or long-term cardiovascular changes caused by static body position have not been studied in detail. Human data suggest that the immediate-term vestibular (otolith organs) activation and the accompanying cardiovascular responses can be maintained for at least $30 \mathrm{~min}$ (Hume and Ray 1999, Shortt and Ray 1997). From our laboratory, it was reported previously that static experimental orthostasis (Raffai et al. 2005) elicits a sustained elevation of arterial blood pressure via increased sympathetic efferent nerve activity in conscious Wistar rats at least for 2 hours. Based on these observations, in the present study we investigated the effect of bilateral vestibular lesions on cardiovascular responses to static orthostatic body position in conscious rats in $1 \mathrm{G}$ environment. We hypothesized, that vestibular lesions suppress both intermediate- and long-term orthostatic responses. In these experiments cardiovascular telemetry (Brockway et al. 1991) was combined with our head-up tilt rat model (Monos et al. 2007) in order to explore the following specific aims:

1. To compare arterial blood pressure and heart rate responses of intact rats maintained in a static headup tilt position for 2 hours with those having bilateral lesions of the vestibular receptors;

2. To compare the cardiovascular responses to static head-up tilt lasting for 2 hours (intermediate) and for 24 hours (long-term);

3. To determine the reproducibility of these responses and to elucidate the role of the vestibular receptor system in the long-term responses.

\section{Methods}

\section{Experimental animals}

Male adult Wistar rats ( $\mathrm{n}=8$, Harlan, Germany) weighing 300-350 $\mathrm{g}$ were used for the experiments. All animals were housed individually with 12-hour light and dark cycles (7 am -7 pm (daytime), 7 pm - 7 am (nighttime), respectively) at $20-22{ }^{\circ} \mathrm{C}$. All animals had free access to standard laboratory rat chow and tap water.

The investigation conforms with the Guide for the Care and Use of Laboratory animals published by the US National Institutes of Health (NIH Publication No. 85-23, revised 1996). Studies were carried out according to the guidelines of Hungarian law on animal protection (243/1998) and approved by the Semmelweis University Committee on the Ethical Use of Experimental Animals (590/99 Rh).

\section{Surgery for telemetric implants}

Radiotelemetric transducer units (Data Sciences International, USA, Type: TL11M2-C50-PXT) were surgically implanted into rats according to a standard operation procedure (Brockway et al. 1991). In brief, rats were anesthetized with sodium-pentobarbital (Sigma, USA, $5 \mathrm{mg} / 100 \mathrm{~g}$ bw, i.p.). Following a midline laparotomy, the catheter of the pressure transducer was 
introduced into the abdominal aorta and fixed with a tissue adhesive. Bipolar ECG leads were tunneled subcutaneously into the Lead II position, and fixed with surgical sutures.

\section{Experimental design and protocol}

During a 10-day recovery period, all rats $(n=8)$ were conditioned to both the tubular tilt cages and the tilting procedures. Familiarization of the rats with these procedures was confirmed, as they entered the cages without exhibiting any avoidance reaction and by exhibiting quiescent behavior while in the cages.

Following the recovery period, arterial blood pressure was sampled at $500 \mathrm{~Hz}$ and recorded continuously using Dataquest A.R.T. data acquisition system (Data Sciences International, USA). This continuous arterial blood pressure record was used to compute mean arterial blood pressure and heart rate variables every 5 seconds.

Intermediate-term orthostatic test was performed on rats that were placed into transparent tiltable tubular cages and restricted from longitudinal locomotion. First, the test cages were set in horizontal position for $40 \mathrm{~min}$, and the last $10 \mathrm{~min}$ quasi-steady state was used as horizontal control record. Starting from the horizontal position, short-term orthostatic test was carried out by positioning the cages with the rats into a $45^{\circ}$ head-up for $120 \mathrm{~min}$. At the end of these tests, rats were returned to the horizontal position for a further 10-min period.

The effect of long-term orthostasis was examined using rats in identical cages as described above. In this case, rats were allowed to longitudinal locomotion along the whole length $(60 \mathrm{~cm})$ of the tubular cages, but they were not able to reverse direction. Chow and tap water were available at the upper edge of the cages. Under these conditions, a 24-hour horizontal control record was made, and then the rats were set in head-up tilt position for an additional 24-hour observation period. Either the last hour of the horizontal record preceding tilt period or the corresponding horizontal values (daytime, nighttime and 24 hours) were used as horizontal control for the long-term head-up tilt responses.

During tilt tests, no head or body fixation was applied, in order to avoid non-specific and nongravitational direct immobilization stress, which can substantially influence the arterial pressure and/or heart rate (Irvine et al. 1997, McDougall et al. 2005).
Hydrostatic component of arterial blood pressure measurement

The 45-degree head-up tilting caused a calculated $3.8 \mathrm{~mm} \mathrm{Hg}$ hydrostatic arterial pressure increment because of the distance between the zero reference level of blood pressure measurement and the tip of the arterial catheter in the abdominal aorta. Corrections were not made, because the effect of gravity in "closed" systems like circulation is more complex and can induce e.g. adaptive changes (Hicks and Badeer 1992).

\section{Lesion of the labyrinth receptor system}

A combined microsurgical-chemical technique, similar to that developed by Matsuda et al. (2004) was performed to produce labyrinthectomy on the 8 rats used before. In detail, the posterior lobe of the glandula parotis had been elevated from its base and the mastoid bulla was exposed, being careful not to damage the facial nerve branches. A 3-4 mm diameter hole was drilled into the posterolateral-medial wall of the mastoid bulla. Then the bony prominence induced by the cochlea on the medial wall of the middle ear was drilled. The endolymph and perilymph were removed by rinsing with saline and concentrated ethanol. Finally, a sodium arsenite $(50 \mu \mathrm{M})$ solution was injected into the labyrinthine cavity. Lesion of the vestibular system was verified by neurological symptoms (typical insecure head and body position, gait of the animals) and by histological examination of decalcified specimens. Both short- and intermediate-term tilt protocols were repeated on 6 out of the 8 rats after a 10-day recovery period following the surgery without complications.

\section{Statistical analysis}

Variables were pooled within regular or specific intervals of the records of intermediate-term (for every $10 \mathrm{~min}$ or for $120 \mathrm{~min}$ of tilts) and long-term (for every hour or for daytime, nighttime, and 24-hour periods) to provide individual data points. These individual data points were used for statistical evaluation of the results which are given either as means \pm S.E.M. or as changes (expressed as $\Delta$ mean \pm S.E.M.) relative to the corresponding horizontal value(s) of intermediate and long-term tilts. One-way repeated measures ANOVA was used for data derived from the intermediate and long-term tests. Dunnett's pairwise multiple comparison procedure was used to test significant differences from horizontal control data. Student t-test was applied when comparing variables between two experimental groups (intact vs. labyrinthectomized). $\mathrm{P}<0.05$ was considered statistically significant. 
Table 1. Summary of horizontal control (HOR) arterial blood pressure and heart rate parameters of intact and labyrinthectomized rats preceding intermediate-term head-up experiments.

\begin{tabular}{lllllll}
\hline & \multicolumn{5}{c}{ Intact } & \multicolumn{3}{c}{ Labyrinthectomized } \\
\cline { 2 - 6 } & $\mathbf{n}$ & $\begin{array}{l}\text { Arterial blood } \\
\text { pressure (mm Hg) }\end{array}$ & $\begin{array}{l}\text { Heart rate } \\
\text { (BPM) }\end{array}$ & $\mathbf{n}$ & $\begin{array}{l}\text { Arterial blood } \\
\text { pressure (mm Hg) }\end{array}$ & $\begin{array}{l}\text { Heart rate } \\
\text { (BPM) }\end{array}$ \\
\hline HOR & 8 & $111.1 \pm 2.0$ & $411.9 \pm 13.8$ & 6 & $105.0 \pm 5.3$ & $393.5 \pm 11.5$ \\
\hline
\end{tabular}

Data are given as mean \pm SEM.

\section{Results}

Horizontal (control) variables in intermediate-term tilt experiments - effect of vestibular lesion

Average values of 10-min steady-state horizontal control arterial pressure and heart rate data in studies for intermediate-term head-up tilt in intact and labyrinthectomized rats are shown in Table 1. Vestibular lesions did not influence significantly these variables preceding intermediate-term head-up tilt experiments.

Effect of vestibular lesion on cardiovascular responses during intermediate-term head-up tilt

As shown in Figure 1, intermediate-term (120 min) head-up tilt induced a sustained elevation of arterial blood pressure by $8.3 \pm 0.9 \mathrm{~mm} \mathrm{Hg}(5-13.7 \mathrm{~mm} \mathrm{Hg})$ without influencing heart rate significantly (10 \pm 12 BPM (1.6-38.8 BPM)). Such blood pressure responses during intermediate-term head-up tilt were eliminated in rats deprived of vestibular inputs (Figure 1), as arterial blood pressure values remained close to horizontal controls $(2 \pm 1.5 \mathrm{~mm} \mathrm{Hg}(-0.7-6.1 \mathrm{mmHg}))$. Head-up tilt tended to decrease heart rate transiently in labyrinthectomized animals, but similarly to intact rats, it did not cause a significant reduction compared to the horizontal control in the 120 min observation period $(-12 \pm 10.8$ BPM (-34.5-7.1 BPM)). The above results were confirmed by comparison of arterial blood pressure and heart rate changes between intact and labyrinthectomised rats. Namely, blood pressure elevation was completely eliminated, while transient heart rate reduction was seen in vestibuloectomized rats compared to intact rats (Fig. 1). It is remarkable, that no significant differences were found in arterial blood pressure changes at the initial 30-min phase of head-up tilt.

Horizontal (control) variables in long-term tilt experiments - effect of vestibular lesion

Arterial blood pressure and heart rate data measured in horizontal body position for 24 hours

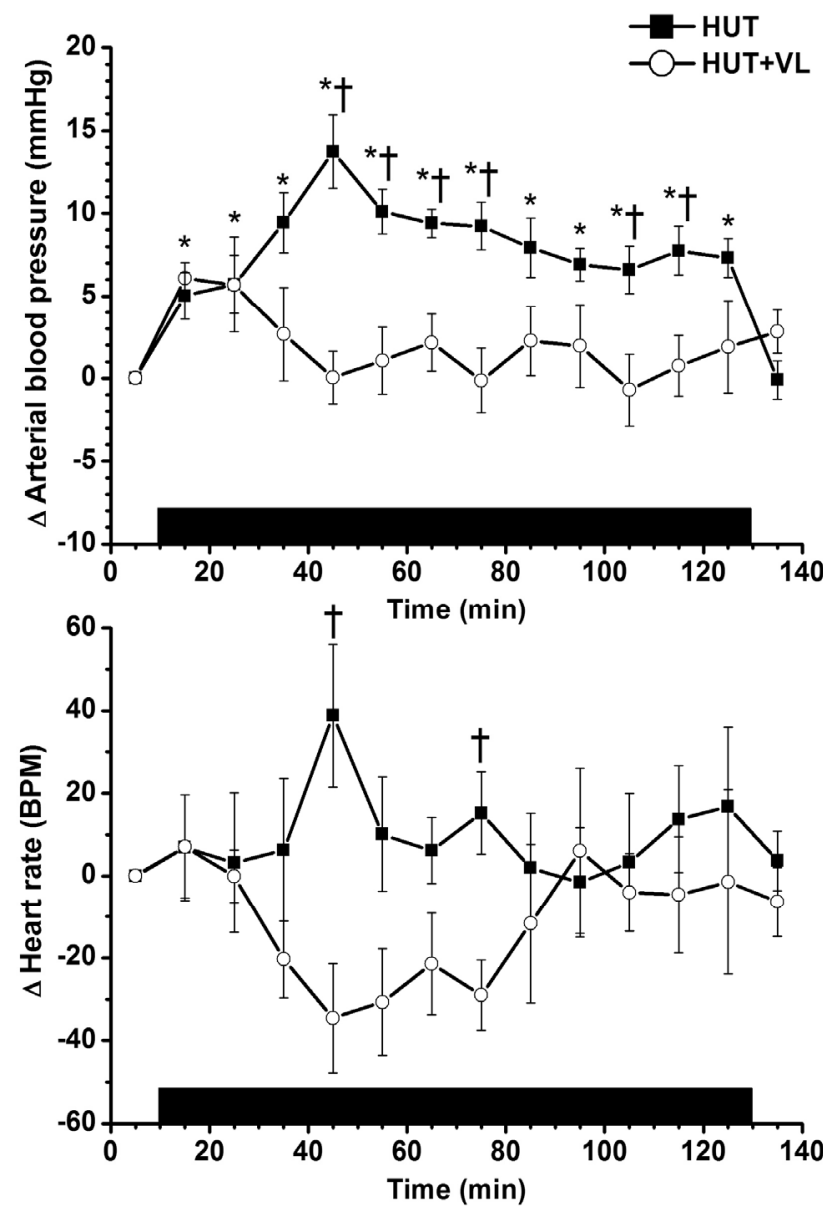

Fig. 1. Changes in arterial blood pressure and heart rate relative to horizontal control values (see HOR in Table 1.) during intermediate-term (120 min) head-up tilt (HUT) experiments in intact rats and in rats with vestibular lesions $(+V L)$. Experimental protocol as follows: horizontal (not labeled), head-up tilt period is indicated with filled bands. Data are given as $\Delta$ mean \pm SEM. Statistical difference $(P<0.05)$ from horizontal controls or between experimental groups (intact vs. $+\mathrm{VL}$ ) is indicated by * or $\dagger$, respectively.

(pooled for daytime, nighttime and 24-hour periods) preceding long-term head-up tilt in intact and labyrinthectomized rats are summarized in Table 2. Heart rate was significantly less in rats with vestibular lesion compared to that of intact rats, while average blood 
Table 2. Summary of horizontal control (HOR), and head-up tilt (HUT) arterial blood pressure and heart rate parameters of intact and labyrinthectomized rats preceding intermediate-term head-up experiments pooled for daytime, nightime, and 24-hour periods.

\begin{tabular}{lllll}
\hline & \multicolumn{2}{l}{ Intact $(\mathbf{n}=\mathbf{8})$} & \multicolumn{2}{l}{ Labyrinthectomized $(\mathbf{n}=\mathbf{6})$} \\
\cline { 2 - 5 } & $\begin{array}{l}\text { Arterial blood } \\
\text { pressure }(\mathbf{m m H g})\end{array}$ & $\begin{array}{l}\text { Heart rate } \\
\mathbf{( B P M )}\end{array}$ & $\begin{array}{l}\text { Arterial blood } \\
\text { pressure }(\mathbf{m m H g})\end{array}$ & $\begin{array}{l}\text { Heart rate } \\
(\mathbf{B P M})\end{array}$ \\
\hline HOR Daytime & $112.9 \pm 2.1$ & $393.9 \pm 4.6$ & $108.6 \pm 4.1$ & $361.4 \pm 13.5 \dagger$ \\
HOR Nighttime & $115.4 \pm 1.9 \S$ & $447.9 \pm 5.8 \S$ & $109.9 \pm 4.9$ & $383.1 \pm 10.8 \S \dagger$ \\
HOR 24 hours & $114.1 \pm 2.0$ & $420.9 \pm 4.5$ & $109.3 \pm 4.4$ & $372.3 \pm 12 \dagger$ \\
HUT Daytime & $118.1 \pm 2.0^{*}$ & $422.3 \pm 11.0^{*}$ & $115.1 \pm 5.4^{*}$ & $389.5 \pm 10.2^{*} \dagger$ \\
HUT Nighttime & $121.3 \pm 2.5 \S^{*}$ & $462.2 \pm 7.0 \S^{*}$ & $111.5 \pm 8.0$ & $423.8 \pm 8.7 \S^{*} \dagger$ \\
HUT 24 hours & $119.7 \pm 2.2^{*}$ & $443.1 \pm 8.2^{*}$ & $113.3 \pm 6.6$ & $406.7 \pm 9.2 * \dagger$ \\
\hline
\end{tabular}

Data are given as mean \pm SEM. $\S, *$, and + statistically different $(P<0.05)$ from corresponding daytime, HOR and intact, respectively.

pressures was not influenced by vestibular lesion. However, diurnal variation of arterial blood pressure was eliminated in horizontal position in vestibuloectomized rats.

Effect of vestibular lesion on cardiovascular responses to long-term head-up tilt

As an effect of 24-hour head-up tilt, arterial blood pressure progressively increased in intact rats from $2.7 \pm 0.6 \mathrm{~mm} \mathrm{Hg}$ (beginning of the tilt) up to $8.8 \pm 1.5$ $\mathrm{mm} \mathrm{Hg}$ when compared to the preceding horizontal values (0-1 hour, Fig. 2). Consequently, daytime, nighttime and 24-hour blood pressure were significantly increased by $5.2 \pm 0.6 \mathrm{~mm} \mathrm{Hg}, 5.9 \pm 1.1 \mathrm{~mm} \mathrm{Hg}$, and $5.6 \pm 0.7 \mathrm{~mm} \mathrm{Hg}$, respectively (Table 2). As shown in Figure 2, after an initial blood pressure increase vestibular lesion led to attenuated blood pressure responses in the further part of the tilt. Such a biphasic response was reflected by the increased daytime (6.4 \pm 1.9 $\mathrm{mm} \mathrm{Hg})$, but decreased nighttime $(1.6 \pm 3.4 \mathrm{~mm} \mathrm{Hg})$ and 24-hour (4 $\pm 2.2 \mathrm{~mm} \mathrm{Hg})$ blood pressure in labyrinthectomized rats (Table 2).

Long term head-up tilt lead to daytime (intact/labyrintectomized, $\quad 28.4 \pm 9.7 / 28.2 \pm 6.8 \quad$ BPM), nighttime (14.3 $\pm 3 / 40.6 \pm 4.3 \mathrm{BPM})$ and 24-hour (22.2 $\pm 6 /$ $34.4 \pm 5.4$ BPM) heart rate increases in both intact and labyrinthectomized animals (Figure 2, Table 2).

\section{Discussion}

The major finding of this work is that blood pressure rise in response to intermediate-term (120 min) experimental orthostasis (head-up tilt) is completely eliminated and heart rate is transiently decreased by removal of the vestibular receptor inputs. Furthermore, long-term (24 hours) head-up tilt results in sustained arterial blood pressure elevation and tachycardia. Labyrinthectomy results in biphasic arterial blood pressure response (initial rise followed by blood pressure reduction) to long-term head-up without altering the tachycardic response. These results support our hypothesis that the vestibular system may contribute to maintain both intermediate- and long-term orthostatic tolerance in conscious rats. In addition, our experimental results indicate that the vestibular system may also play an essential role in longer ( $>5 \mathrm{~min})$ cardiovascular adaptation.

Role of the vestibular receptors in intermediate-term orthostatic cardiovascular responses

As shown earlier, blood pressure elevation induced by intermediate-term orthostasis is elicited by sympathetic activation (Raffai et al. 2005). In the present study, we found that vestibular lesions completely eliminated blood pressure responses without leading to the serious cardiovascular symptoms of orthostatic intolerance (hypotension, cardioacceleration) in conscious rats, unlike in anesthetized cats (Doba and Reis 1974, Woodring et al. 1997). Our results also provide experimental evidence for a direct contribution of the vestibular apparatus to the control of intermediate-term arterial blood pressure responses induced by static changes of body position. Similarly to our results, other investigators (Jian et al. 1999, Mori et al. 2005) demonstrated that bilateral vestibular lesion alters orthostatic responses leading to arterial blood pressure instability in conscious cats during nose up tilt. Our observations, made under $1 \mathrm{G}$ conditions, are also in 

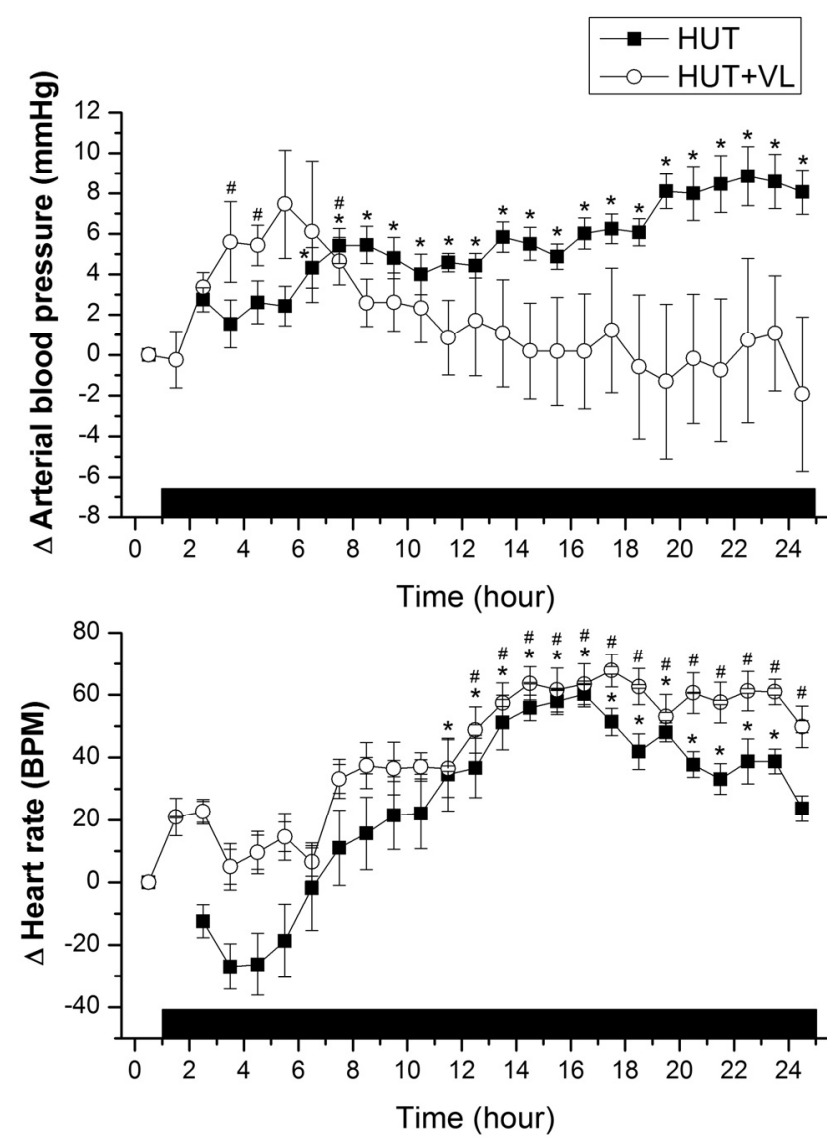

Fig. 2. Changes in arterial blood pressure and heart rate relative to the corresponding horizontal control values in intact rats (114.2 $\pm 1.9 \mathrm{~mm} \mathrm{Hg} / 417.3 \pm 9.1 \mathrm{BPM})$ and rats with vestibular lesion (+VL; $111.5 \pm 6.1 \mathrm{~mm} \mathrm{Hg} / 363.8 \pm 7.4 \mathrm{BPM}+$ ) during longterm (24-h) head-up tilt (HUT). Experimental protocol as follows: horizontal (not labeled), head-up tilt period is indicated with filled bands. Data are given as $\Delta$ mean \pm SEM. Statistical difference $(P<0.05)$ from horizontal controls in intact and labyrinthectomized rats is indicated by $*$ or \#, respectively.

good agreement with the results of studies conducted under shorter experimental microgravity and hypergravity conditions where both the pressor and the renal sympathetic nerve activity responses were attenuated by vestibular lesion (Gotoh et al. 2004, Tanaka et al. 2006).

In the present study, there was no difference between intact and labyrinthectomized rats in the rapidly developing arterial blood pressure responses to the initial phases of head-up $(30 \mathrm{~min})$ tilt, indicating that early blood pressure responses might not be influenced by the lack of vestibular inputs in the rat. This response pattern may be attributed to gravity related reflexes other than vestibulosympathetic reflex activated by sudden changes of arterial blood pressure due to tilting. Following this phase, intact rats responded with a sustained and stable rise of arterial pressure, which did not show any restoration or adaptation during the 24-hour observation period. In contrast, elimination of vestibular inputs eliminated such blood pressure elevations. These results provide evidence that vestibular inputs may play a role not only in the short latency and rapid feed-forward control of arterial blood pressure (Voustianiouk et al. 2006), but also in longer arterial pressure control during sustained gravitational stress, as previously indicated in human studies (Hume and Ray 1999, Shortt and Ray 1997). Because of the additive nature of the vestibulosympathetic reflex and the baroreflex (Ray 2000, Shortt and Ray 1997), the lack of blood pressure elevation in labyrinthectomized, but baroreflex intact rats might be due to the absence of converging vestibular signals in the rostral ventrolateral medulla (Yates et al. 1993, Yates et al. 2000).

Role of the vestibular system in long-term orthostatic cardiovascular responses

In contrast to the intermediate-tilt experiments, initial blood pressure elevation to long-term orthostasis was delayed by 2-4 hours in both experimental groups. Such a delayed blood pressure responses might be attributed to the 24-hour confined horizontal body position, which preceded the tilt reducing cardiovascular responsiveness to orthostatic stress. Similar delayed blood pressure responsiveness was not observed either after 40 min horizontal body position (intermediate-term tilt) in this or after 7 day head-down tilt in our previous study (Raffai et al. 2009). Blood pressure elevation was accompanied by heart rate increase in the long-term tilts. Similar to intermediate-term cardiovascular responses (Raffai et al. 2005), we assume that the rise in arterial blood pressure and heart rate that we observed in the long-term tilt experiments is caused by coordinated activation of baroreflex and different gravity-related receptor mechanisms (vestibular, proprioceptive, visual, visceral, and cutaneous inputs), which induce a compensatory readjustment of the cardiovascular system. Activation of this complex receptor system ultimately leads to a compensatory pressor response that can be explained by sympathetic activation and/or parasympathetic withdrawal. This assumption is supported by human studies where stimulation of vestibular otolith organs increases sympathetic output via the vestibulosympathetic reflex, which works independently of the baroreflex (Ray 2000) or skeletal muscle reflex(es) (Ray 2001). However, the final effect of these reflexes is additive (Ray 2000, Ray 2001). In addition to such autonomic readjustments, elevated plasma concentrations 
of stress hormones are likely to contribute to maintaining cardiovascular responses, after some delay. This hypothesis is in accordance with our earlier observations (Monos et al. 2007) and with those of Assenmacher et al. (1995) demonstrating that orthostatic restraint induces a transient elevation of stress hormone levels (e.g. ACTH, corticosterone). Heart rate elevations in our study suggest an impairment of heart rate decreasing mechanisms, as the reduced baroreflex sensitivity (unpublished observation).

Vestibular lesions resulted in an initial blood pressure rise followed by blood pressure reduction in head-up tilting while tachycardia was not influenced by vestibuloectomy. According to our knowledge, this is the first experimental evidence that vestibular inputs play a role in blood pressure responses in orthostatic body positions maintained for 24 hours. It remains to be determined that why vestibuloectomy, besides attenuating orthostatic blood pressure responses, also eliminates diurnal variations of arterial blood pressure both in horizontal and head-up tilt position. It is also remarkable that in contrast to the well-known adaptive plasticity of the vestibular system (Jian et al. 1999, Mori et al. 2005, Wilson et al. 2006), the lack of vestibular inputs can still influence both intermediate- and long-term gravitational responses following vestibuloectomy and the subsequent recovery period.

\section{Non-specific stress and gravitational stress}

In the present study, we examined arterial blood pressure and heart rate responses in static body positions, without exposing the rats to dynamic forces (linear or angular acceleration) experimentally. Linear or angular acceleration can occasionally occur during normal activity of the rats, but we assume that the probability of these events is the same in intact and labyrinthectomized rats at an identical body axis (horizontal, or $45^{\circ}$ head-up). The absence of substantial non-specific (nongravitational) stress was confirmed by the calm behavior of the rats without showing any avoidance reactions during the sustained tilt tests. Furthermore, a horizontal body position of $120 \mathrm{~min}$ (Raffai et al. 2005, Raffai et al. 2006) or longer $(24 \mathrm{~h})$ did not lead to an elevation of arterial blood pressure or heart rate itself. In our studies, rats were not exposed to additional non-specific or nongravitational (e.g. direct immobilization, startle stimulus etc.) stress that can substantially influence the basic cardiovascular variables (Irvine et al. 1997, McDougall et al. 2005). Stress reaction caused by visual, visceral, and cutaneous inputs could also be taken into account, but the exposure of intact and labyrinthectomized rats to these sensory inputs was essentially the same. Thus, we have good reasons to conclude that instead of environmental factors, the sustained arterial blood pressure and heart rate responses to tilting were elicited specifically by changes in body position.

In conclusion, the vestibular system may play a role in maintaining arterial blood pressure at orthostatic body position providing sustained inputs for the appropriate cardiovascular readjustments for several hours. This role is reflected by the development of sustained arterial pressure elevation due to intermediateand long-term gravitational stress and tachycardia in the latter case. Lack of vestibular inputs under these conditions does not lead to orthostatic arterial hypotension, as one could expect, it is obviously prevented by other cardiovascular reflexes.

\section{Conflict of Interest}

There is no conflict of interest.

\section{Acknowledgements}

This work was supported by Hungarian Grants: OTKA T042670/2003 and ETT 128/2006. We are grateful to Dr. Julian H. Lombard for his help in editing the manuscript. Authors are indebted to Drs. Sándor Farkas and Márk Kollai for their continuous support.

\section{References}

ASSENMACHER I, MEKAOUCHE M, MAUREL D, BARBANEL G, GIVALOIS L, BOISSIN J, MALAVAL F, IXART G: Chronic orthostatic and antiorthostatic restraint induce neuroendocrine, immune and neurophysiologial disorders in rats. Acta Astronaut 36: 545-558, 1995.

BALABAN CD, BERYOZKIN G: Vestibular nucleus projections to nucleus tractus solitarius and the dorsal motor nucleus of the vagus nerve: potential substrates for vestibulo-autonomic interactions. Exp Brain Res 98: 200$212,1994$. 
BROCKWAY BP, MILLS PA, AZAR SH: A new method for continuous chronic measurement and recording of blood pressure, heart rate and activity in the rat via radio-telemetry. Clin Exp Hypertens 13: 885-895, 1991.

DOBA N, REIS DJ: Role of the cerebellum and the vestibular apparatus in regulation of orthostatic reflexes in the cat. Circ Res 40: 9-18, 1974.

GOTOH TM, FUJIKI N, MATSUDA T, GAO S, MORITA H: Roles of baroreflex and vestibulosympathetic reflex in controlling arterial blood pressure during gravitational stress in conscious rats. Am J Physiol 286: R25-R30, 2004.

HICKS JW, BADEER HS: Gravity and the circulation: "open" vs. "closed" systems. Am J Physiol 262: R725-R732, 1992.

HUME KM, RAY CA: Sympathetic responses to head-down rotations in humans. J Appl Physiol 86: 1971-1976, 1999.

IRVINE RJ, WHITE J, CHAN R: The influence of restraint on blood pressure in the rat. $J$ Pharmacol Toxicol Methods 38: 157-162, 1997.

JIAN BJ, COTTER LA, EMANUEL BA, CASS SP, YATES BJ: Effects of bilateral vestibular lesions on orthostatic tolerance in awake cats. $J$ Appl Physiol 86: 1552-1560, 1999.

JIAN BJ, SHINTANI T, EMANUEL BA, YATES BJ: Convergence of limb, visceral, and vertical semicircular canal or otolith inputs onto vestibular nucleus neurons. Exp Brain Res 144: 247-257, 2002.

KERMAN IA, MCALLEN RM, YATES BJ: Patterning of sympathetic nerve activity in response to vestibular stimulation. Brain Res Bull 53: 11-16, 2000.

MATSUDA T, GOTOH T M, TANAKA K, GAO S, MORITA H: Vestibulosympathetic reflex mediates the pressor response to hypergravity in conscious rats: contribution of the diencephalon. Brain Res 1028: 140-147, 2004.

MCDOUGALL SJ, LAWRENCE AJ, WIDDOP RE: Differential cardiovascular responses to stressors in hypertensive and normotensive rats. Exp Physiol 90: 141-150, 2005.

MONOS E, LÓRÁNT M: Vestibular control of the cardiovascular system. Orv Hetil 139: 1851-1855, 1998.

MONOS E, RAFFAI G, DÖRNYEI G, NÁDASY G, FEHÉR E: Structural and functional responses of extremity veins to long-term gravitational loading or unloading - lessons from animal systems. Acta Astronaut 60: 406-414, 2007.

MORI RL, COTTER LA, ARENDT HE, OLSHESKI CJ, YATES BJ: Effects of bilateral vestibular nucleus lesions on cardiovascular regulation in conscious cats. $J$ Appl Physiol 98: 526-533, 2005.

RAFFAI G, MÉSZÁROS M, KOLLAI M, MONOS E, DÉZSI L: Experimental orthostasis elicits sustained hypertension, which can be prevented by sympathetic blockade in the rat. J Cardiovasc Pharmacol 45: 354$361,2005$.

RAFFAI G, KOCSIS L, MÉSZÁROS M, MONOS E, DÉZSI L: Inverse-orthostasis may induce elevation of blood pressure due to sympathetic activation. J Cardiovasc Pharmacol 47: 287-294, 2006.

RAFFAI G, CSEKO C, KOCSIS L, DEZSI L, MONOS E: Does long-term experimental antiorthostasis lead to cardiovascular deconditioning in the rat? Physiol Res 58: 57-67, 2009.

RAY CA: Interaction of the vestibular system and baroreflexes on sympathetic nerve activity in humans. Am J Physiol 279: H2399-H2404, 2000.

RAY CA: Interaction between vestibulosympathetic and skeletal muscle reflexes on sympathetic activity in humans. J Appl Physiol 90: 242-247, 2001.

RAY CA, CARTER JR: Vestibular activation of sympathetic nerve activity. Acta Physiol Scand 177: 313-319, 2003.

RAY CA, HUME KM: Neck afferents and muscle sympathetic activity in humans: implications for the vestibulosympathetic reflex. $J$ Appl Physiol 84: 450-453, 1998a.

RAY CA, HUME KM, STEELE SL: Sympathetic nerve activity during natural stimulation of horizontal semicircular canals in humans. Am J Physiol 275: R1274-R1278, $1998 \mathrm{~b}$.

SHORTT TL, RAY CA: Sympathetic and vascular responses to head-down neck flexion in humans. Am J Physiol 272: H1780-H1784, 1997.

TANAKA K, GOTOH TM, AWAZU C, MORITA H: Roles of the vestibular system in controlling arterial pressure in conscious rats during a short period of microgravity. Neurosci Lett 397: 40-43, 2006. 
VOUSTIANIOUK A, KAUFMANN H, DIEDRICH A, RAPHAN T, BIAGGIONI I, MACDOUGALL H, OGORODNIKOV D, COHEN B: Electrical activation of the human vestibulo-sympathetic reflex. Exp Brain Res 171: 251-261, 2006.

WILSON TD, COTTER LA, DRAPER JA, MISRA SP, RICE CD, CASS SP, YATES BJ: Vestibular inputs elicit patterned changes in limb blood flow in conscious cats. J Physiol Lond 575: 671-684, 2006.

WOODRING SF, ROSSITER CD, YATES BJ: Pressor response elicited by nose-up vestibular stimulation in cats. Exp Brain Res 113: 165-168, 1997.

YATES BJ, BRONSTEIN AM: The effects of vestibular system lesions on autonomic regulation: observations, mechanisms, and clinical implications. J Vestib Res 15: 119-129, 2005.

YATES BJ, GOTO T, BOLTON PS: Responses of neurons in the rostral ventrolateral medulla of the cat to natural vestibular stimulation. Brain Res 601: 255-264, 1993.

YATES BJ, HOLMES MJ, JIAN BJ: Adaptive plasticity in vestibular influences on cardiovascular control. Brain Res Bull 53: 3-9, 2000. 